\title{
Ratiometric and Water-soluble Fluorescent Zinc Sensor of Carboxamidoquinoline with an Alkoxyethylamino Chain as Receptor
}

\author{
Yu Zhang, ${ }^{\mathrm{a}, \mathrm{b}}$ Xiangfeng Guo, ${ }^{, \mathrm{b}}$ Wanxia Si, ${ }^{\mathrm{b}}$ Lihua Jia, ${ }^{\mathrm{b}}$ and Xuhong Qian, ${ }^{\text {,a,c }}$ \\ ${ }^{a}$ State Key Laboratory of Fine Chemicals, Dalian University of Technology, Dalian 116012, China, \\ ${ }^{\mathrm{b}}$ Key Laboratory of Fine Chemicals (Qiqihar University), College of Heilongjiang Province, Qiqihar 161006, China, \\ 'Shanghai Key Laboratory of Chemical Biology, East China University of Science and Technology, Shanghai 200237, China \\ *Corresponding author:xfguo@163.com; xhqian@ecust.edu.cn
}

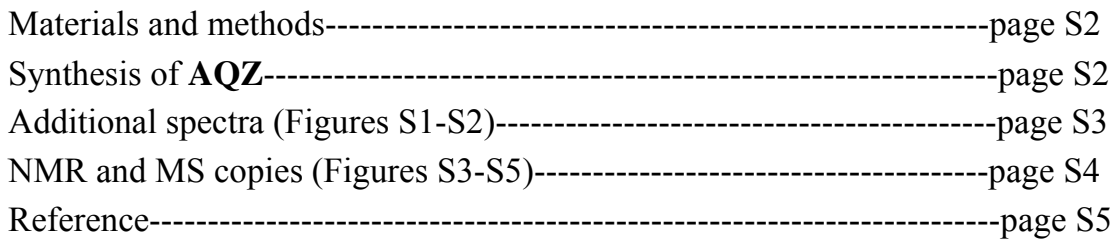




\section{Materials and methods}

All of the solvents were of analytic grade. ${ }^{1} \mathrm{H}-\mathrm{NMR}$ and ${ }^{13} \mathrm{C}-\mathrm{NMR}$ were measured on a Bruker AV-400 spectrometer with chemical shifts reported as ppm (in $\mathrm{CDCl}_{3}$, TMS as internal standard). MALDI-TOF mass spectra were measured on an ABI Voyager DE-Pro MALDI-TOF spectrometer. IR spectra were obtained on a Nicolet AVATAR-370 FT-IR instrument, and samples were prepared as $\mathrm{KBr}$ pellets. Melting points were determined by an X-6 micro-melting point apparatus and uncorrected. All pH measurements were made with a Sartorius basic pH-meter PB-10. Fluorescence and absorption spectra were recorded on a Hitachi F-4500 fluorescence spectrometer and a Pgeneral TU-1901 UV-vis spectrophotometer. All imaging experiments were performed on an Olympus BX51 fluorescence microscope with excitation at 330-385 $\mathrm{nm}$ and a DP71 color CCD camera for photo collection. The total magnification is $400 \times$.

All of the detections of metal ions were operated at $\mathrm{pH} 7.22$ maintained with tris- $\mathrm{HCl}$ buffer $(0.01 \mathrm{M})$. The stock solution of AQZ was prepared in methanol $(0.001 \mathrm{M})$. The cational solutions were prepared from $\mathrm{NaClO}_{4}, \mathrm{KClO}_{4}, \mathrm{Mg}\left(\mathrm{ClO}_{4}\right)_{2}$, $\mathrm{Ca}\left(\mathrm{NO}_{3}\right)_{2}, \mathrm{Fe}_{2}\left(\mathrm{SO}_{4}\right)_{3}, \mathrm{CoSO}_{4}, \mathrm{NiSO}_{4}, \mathrm{Cu}\left(\mathrm{NO}_{3}\right)_{2}, \mathrm{Zn}\left(\mathrm{NO}_{3}\right)_{2}, \mathrm{AgNO}_{3}, \mathrm{CdSO}_{4}, \mathrm{HgCl}_{2}$ or $\mathrm{Pb}\left(\mathrm{NO}_{3}\right)_{2}$ in distilled water with a concentration of $0.01 \mathrm{M}$, respectively. The volume of cationic stock solution added was less than $100 \mu \mathrm{L}$ to remain the concentration of AQZ unchanged. All fluorescence spectra were recorded at $25{ }^{\circ} \mathrm{C}$ with the excitation of $325 \mathrm{~nm}$. Fluorescence quantum yields were obtained by using quinine bisulphate in $0.1 \mathrm{M} \mathrm{H}_{2} \mathrm{SO}_{4}(\Phi=0.55)$ as a reference. ${ }^{1}$

Yeast (saccharomyces cerevisiae) was obtained from Zhuhai Ziying Biotechnology Co Ltd and used as a cell model. Before use, it was dispersed in YPD medium (1.0\% yeast extract, $4.0 \%$ peptone, $2.0 \%$ glucose, $\left.0.2 \%\left(\mathrm{NH}_{4}\right)_{2} \mathrm{SO}_{4}\right)$ and incubated for $12 \mathrm{~h}$ at $37{ }^{\circ} \mathrm{C}$. For yeast staining, some colonies were selected and incubated with $40 \mu \mathrm{M} \mathrm{AQZ} \mathrm{in} \mathrm{tris-HCl}$ $(0.01 \mathrm{M})$ solution $(\mathrm{pH} 7.22)$ for $1 \mathrm{~h}$ at $37^{\circ} \mathrm{C}$. The sensor solution was then removed and the cells were washed twice with PBS buffer (0.01 M, pH 7.40) to remove free AQZ. AQZ pretreated cells were then diluted with the addition of $\mathrm{ZnSO}_{4}(40$ $\mu \mathrm{M})$ and incubated for another $0.5 \mathrm{~h}$ at $37{ }^{\circ} \mathrm{C}$. The cells for experiments were dropped on glass slides and covered with a cover glass.

\section{Synthesis of AQZ}

Preparation of 2-chloro-N-(quinol-8-yl)acetamide. 2-chloroacetyl chloride (461 $\mathrm{mg}, 2.4 \mathrm{mmol}$ ) was dissolved in chloroform $(5 \mathrm{~mL})$, then added dropwise to a cooled, stirred solution of 8-aminoquinoline $(288 \mathrm{mg}, 2.0 \mathrm{mmol})$ and pyridine (222 $\mathrm{mg}, 2.8 \mathrm{mmol})$ in chloroform $(10 \mathrm{~mL})$ within $1 \mathrm{~h}$, after stirred $2 \mathrm{~h}$ at $\mathrm{rt}$, the mixture was removed under reduced pressure to obtain a white solid, which was purified by silica gel column chromatography using dichloromethane as eluent to afford 2-chloro- $N$-(quinol-8-yl)acetamide. Yield: $353 \mathrm{mg}$ (79.9\%), mp: 131.6 132.6 ${ }^{\circ} \mathrm{C} .{ }^{1} \mathrm{H}-\mathrm{NMR}\left(\mathrm{CDCl}_{3}, 400 \mathrm{MHz}\right.$, TMS): $\delta 10.92(\mathrm{NHCO}, \mathrm{s}, 1 \mathrm{H}), 8.82(\mathrm{~d}, J=2.8 \mathrm{~Hz}, 1 \mathrm{H}), 8.76(\mathrm{~d}, J=6.4 \mathrm{~Hz}, 1 \mathrm{H}), 8.20(\mathrm{~d}, J=8.4 \mathrm{~Hz}, 1 \mathrm{H}), 7.59(\mathrm{~m}, 2 \mathrm{H}), 7.50(\mathrm{~m}$, $1 \mathrm{H}), 4.32\left(\mathrm{COCH}_{2}, \mathrm{~s}, 2 \mathrm{H}\right) \mathrm{ppm}$.

Preparation of AQZ. 2-chloro- $N$-(quinol-8-yl)acetamide (100 mg, $0.45 \mathrm{mmol})$, 2-(2-aminoethoxy)ethanol (746 mg, 4.53 $\mathrm{mmol}), N, N$-diisopropylethylamine $(586 \mathrm{mg}, 4.53 \mathrm{mmol})$ and potassium iodide $(10 \mathrm{mg})$ were added to acetonitrile $(30 \mathrm{~mL})$, after stirred and refluxed for $10 \mathrm{~h}$ under nitrogen atmosphere, the mixture was cooled to $\mathrm{rt}$ and the mixture was removed under reduced pressure to obtain a yellow oil, which was purified by silica gel column chromatography using chloroform/methanol (20:1, v/v) as eluent to afford AQZ. Yield: $110 \mathrm{mg}(83.8 \%) .{ }^{1} \mathrm{H}-\mathrm{NMR}\left(\mathrm{CDCl}_{3}, 400 \mathrm{MHz}, \mathrm{TMS}\right): \delta$ $11.24(\mathrm{NHCO}, \mathrm{s}, 1 \mathrm{H}), 8.86(\mathrm{~d}, J=5.6 \mathrm{~Hz}, 1 \mathrm{H}), 8.81(\mathrm{~d}, J=8.8 \mathrm{~Hz}, 1 \mathrm{H}), 8.16(\mathrm{~d}, J=9.6 \mathrm{~Hz}, 1 \mathrm{H}), 7.53(\mathrm{~m}, 2 \mathrm{H}), 7.46(\mathrm{~m}$, 
$1 \mathrm{H}), 3.74\left(\mathrm{O}-\mathrm{CH}_{2} \mathrm{CH}_{2}-\mathrm{OH}, \mathrm{m}, 4 \mathrm{H}\right), 3.59\left(\mathrm{COCH}_{2}, \mathrm{NHCH}_{2}-\mathrm{CH}_{2}, \mathrm{~m}, 4 \mathrm{H}\right), 2.99\left(\mathrm{CH}_{2} \mathrm{NH}-\mathrm{CH}_{2}, \mathrm{t}, J=4.8 \mathrm{~Hz}, 2 \mathrm{H}\right) \mathrm{ppm} .{ }^{13} \mathrm{C}-$ $\mathrm{NMR}\left(\mathrm{CDCl}_{3}, 100 \mathrm{MHz}\right): \delta 170.82,148.55,138.94,136.30,133.29,128.10,127.33,121.82,121.56,116.70,72.32,70.70$, 61.82, 53.77, 49.43 ppm. MALDI-TOF: $[\mathrm{M}+\mathrm{H}]^{+}$calcd for $\mathrm{C}_{15} \mathrm{H}_{20} \mathrm{~N}_{3} \mathrm{O}_{3}, 290.1499$; found 290.1495. FT-IR (KBr): $v_{\max }$ $3331.09,2927.52,2867.82,1681.82,1529.10,1488.16,1325.10,1121.33,1065.83,826.80,793.48 \mathrm{~cm}^{-1}$.

\section{Additional spectra}

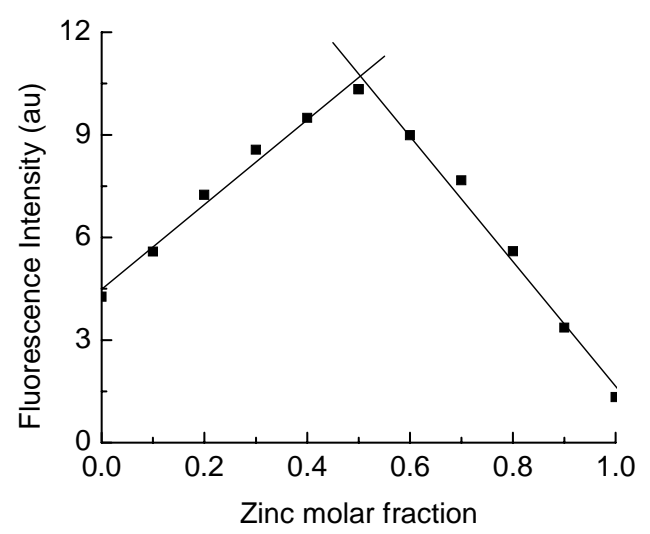

Figure S1. Job's plot for AQZ (forms 1:1 complexes) in tris- $\mathrm{HCl}(0.01 \mathrm{M})$ solution (methanol/water $=1: 9, \mathrm{v} / \mathrm{v}, \mathrm{pH}=7.22)$. The total $[\mathbf{A Q Z}]+\left[\mathrm{Zn}\left(\mathrm{NO}_{3}\right)_{2}\right]=10 \mu \mathrm{M}$.

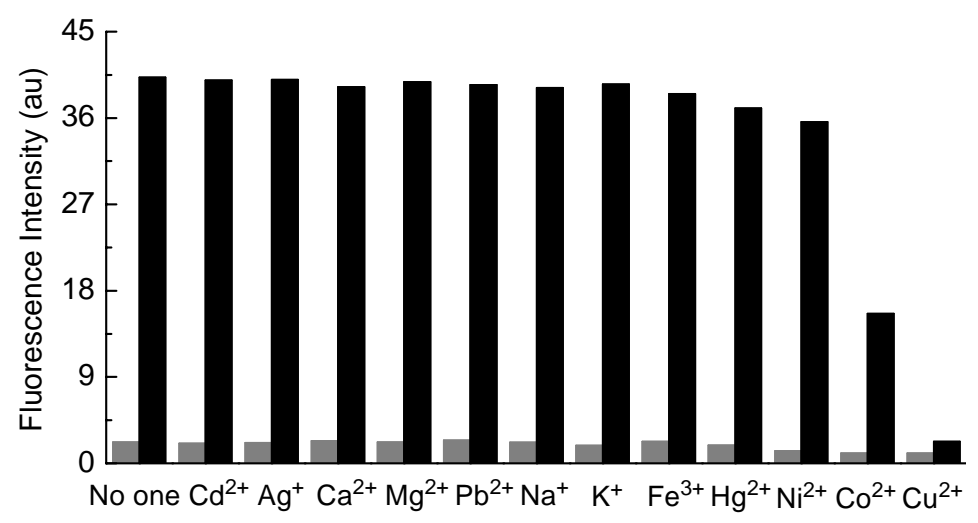

Figure S2. $\mathbf{M}^{\mathrm{n}+}$-selectivity profile of AQZ $(10 \mu \mathrm{M})$ : (grey bars) relative fluorescence intensity of $\mathbf{A Q Z}+1$ equiv $\mathbf{M}^{\mathrm{n}+}$ at 515 $\mathrm{nm}$; (black bars) relative fluorescence intensity of $\mathbf{A Q Z}+1$ equiv $\mathrm{M}^{\mathrm{n}+}$, followed by 1 equiv $\mathrm{Zn}^{2+}$ at $515 \mathrm{~nm}$ in tris- $\mathrm{HCl}(0.01$ M) solution (methanol/water $=1: 9, \mathrm{v} / \mathrm{v}, \mathrm{pH}=7.22)$. 
NMR and MS copies

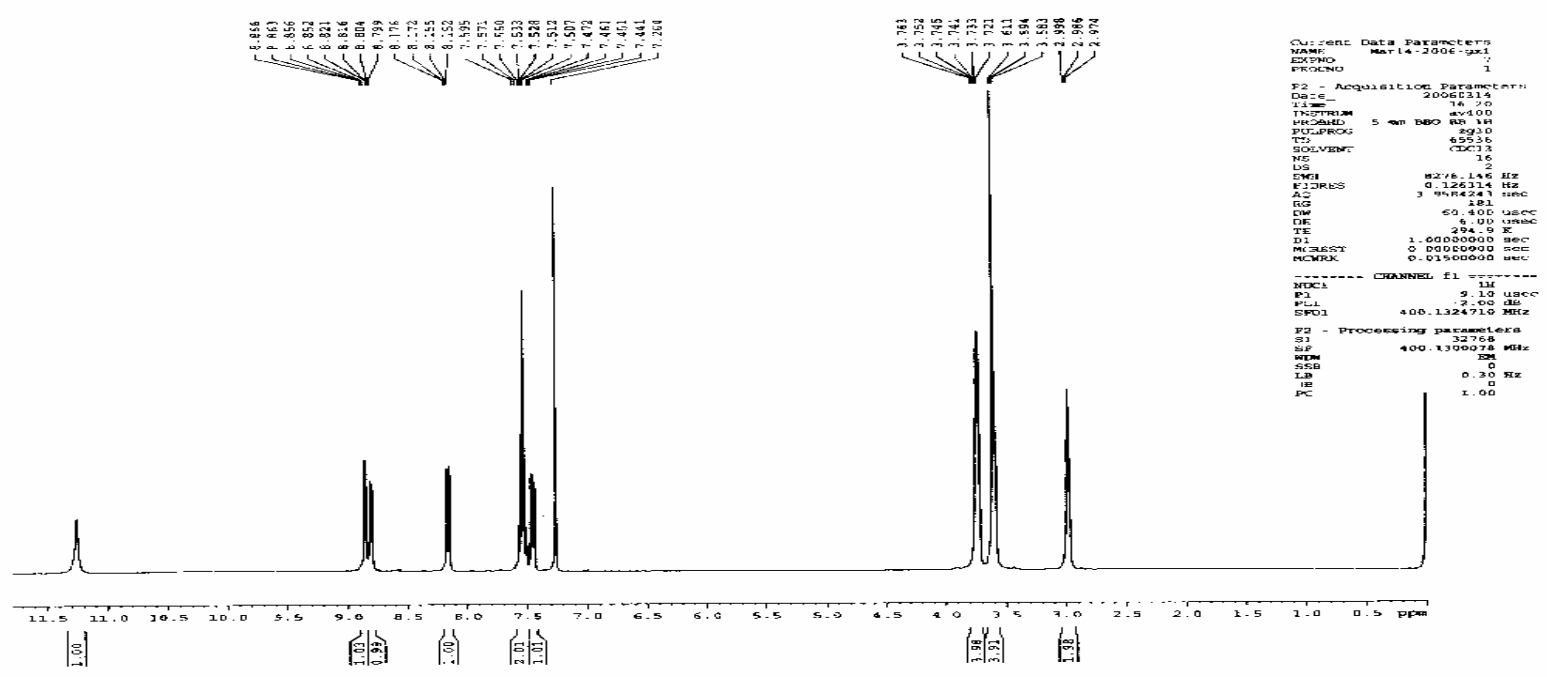

Figure S3. ${ }^{1} \mathrm{H}-\mathrm{NMR}\left(\mathrm{CDCl}_{3}, 400 \mathrm{MHz}\right)$ spectrum of compound AQZ.

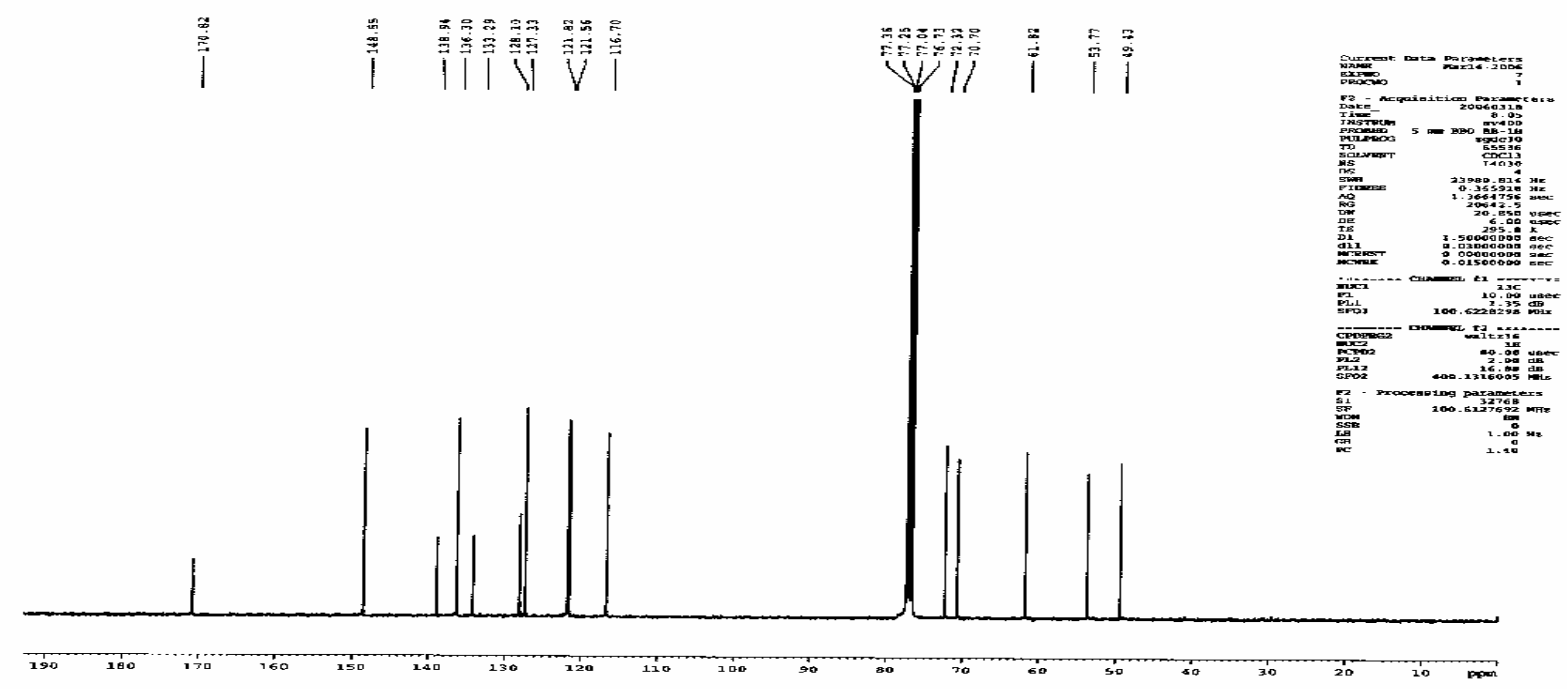

Figure S4. ${ }^{13} \mathrm{C}-\mathrm{NMR}\left(\mathrm{CDCl}_{3}, 100 \mathrm{MHz}\right)$ spectrum of compound AQZ. 


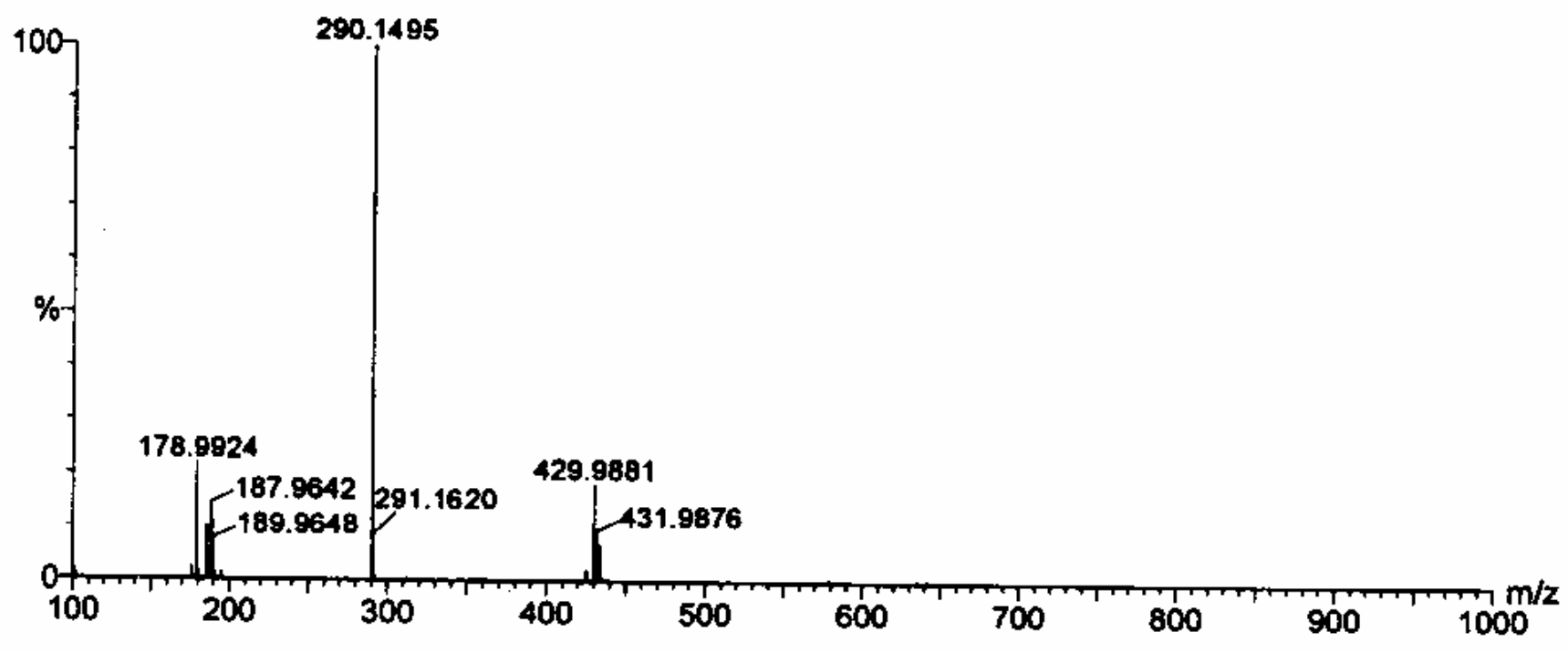

Figure S5. Mass spectrum of compound AQZ.

\section{Reference}

(1) Parker, C. A.; Rees, W. T. Analyst 1960, 85, 587-600. 